\title{
Pseudoneglect in line bisection judgement is associated with a modulation of right hemispheric spatial attention dominance in right-handers
}

\author{
Laure Zago ${ }^{\mathrm{a}, \mathrm{b}, \mathrm{c}, *}$, Laurent Petit ${ }^{\mathrm{a}, \mathrm{b}, \mathrm{c}}$, Gael Jobard ${ }^{\mathrm{a}, \mathrm{b}, \mathrm{c}}$, Julien Hay ${ }^{\mathrm{a}, \mathrm{b}, \mathrm{c}, 1}$, \\ Bernard Mazoyer ${ }^{\mathrm{a}, \mathrm{b}, \mathrm{c}}$, Nathalie Tzourio-Mazoyer ${ }^{\mathrm{a}, \mathrm{b}, \mathrm{c}}$, Hans-Otto Karnath ${ }^{\mathrm{d}}$, Emmanuel Mellet ${ }^{\mathrm{a}, \mathrm{b}, \mathrm{c}}$ \\ a Université de Bordeaux, Institut des Maladies Neurodégénératives, Groupe d'Imagerie Neurofonctionnelle, UMR 5293, F-33000 Bordeaux, France \\ b CNRS, Institut des Maladies Neurodégénératives, Groupe d'Imagerie Neurofonctionnelle, UMR 5293, F-33000 Bordeaux, France \\ c CEA, Institut des Maladies Neurodégénératives, Groupe d'Imagerie Neurofonctionnelle, UMR 5293, F-33000 Bordeaux, France \\ d Center of Neurology, Division of Neuropsychology, Hertie Institute of Clinical Brain Research, University of Tübingen, 72076 Tübingen, Germany
}

\section{A R T I C L E I N F O}

\section{Keywords:}

Spatial attention

Hemispheric lateralization

Pseudoneglect

Spatial neglect

Line bisection

FMRI

Human

\begin{abstract}
A B S T R A C T
The objective of this study was to validate a line bisection judgement (LBJ) task for use in investigating the lateralized cerebral bases of spatial attention in a sample of 51 right-handed healthy participants. Using functional magnetic resonance imaging (fMRI), the participants performed a LBJ task that was compared to a visuomotor control task during which the participants made similar saccadic and motoric responses. Cerebral lateralization was determined using a voxel-based functional asymmetry analysis and a hemispheric functional lateralization index (HFLI) computed from fMRI contrast images. Behavioural attentional deviation biases were assessed during the LBJ task and a "paper and pencil" symbol cancellation task (SCT). Individual visuospatial skills were also evaluated. The results showed that both the LBJ and SCT tasks elicited leftward spatial biases in healthy subjects, although the biases were not correlated, which indicated their independence. Neuroimaging results showed that the LBJ task elicited a right hemispheric lateralization, with rightward asymmetries found in a large posterior occipito-parietal area, the posterior calcarine sulcus (V1p) and the temporo-occipital junction (TOJ) and in the inferior frontal gyrus, the anterior insula and the superior medial frontal gyrus. The comparison of the LBJ asymmetry map to the lesion map of neglect patients who suffer line bisection deviation demonstrated maximum overlap in a network that included the middle occipital gyrus (MOG), the TOJ, the anterior insula and the inferior frontal region, likely subtending spatial LBJ bias. Finally, the LBJ task-related cerebral lateralization was specifically correlated with the LBJ spatial bias but not with the SCT bias or with the visuospatial skills of the participants. Taken together, these results demonstrated that the LBJ task is adequate for investigating spatial lateralization in healthy subjects and is suitable for determining the factors underlying the variability of spatial cerebral lateralization.
\end{abstract}

\section{Introduction}

Hemispheric specialization (HS) is a fundamental principle in the functional organization of the human brain (Hervé et al., 2013). In most humans, the left hemisphere is specialized for language, praxis and motor control of the dominant hand, whereas the right hemisphere is more dedicated to the control of visuospatial skills and spatial attention (Heilman et al., 1993; Karnath and Rorden, 2012; Kinsbourne, 1970; Mazoyer et al., 2014; Mesulam, 1999). However, this is not an invariable principle. For example, there are occasional patients with aphasia after right hemispheric lesions or with neglect after left hemispheric lesions (Coppens et al., 2002; Dronkers and
Knight, 1989; Suchan and Karnath, 2011). Although the variability of cerebral lateralization for language has been extensively studied in healthy participants (Josse and Tzourio-Mazoyer, 2004; Tzourio et al., 1998), right hemispheric dominance has been considered a poor relation of hemispheric specialization. To our knowledge, there is no "gold standard" for assessing hemispheric spatial dominance, such as the Wada test (Wada and Rasmussen, 1960) that remains the gold standard for assessing individual language lateralization in preoperative patients. More recently, functional magnetic resonance imaging (fMRI) has been shown to be a valid non-invasive alternative to Wada testing to assess functional language lateralization (see for reviews Binder, 2011; Dym et al., 2011). Regarding the cerebral dominance of

\footnotetext{
* Corresponding author at: Université de Bordeaux, Institut des Maladies Neurodégénératives, Groupe d’Imagerie Neurofonctionnelle, UMR 5293, F-33000 Bordeaux, France. E-mail address: laure.zago@u-bordeaux.fr.

${ }^{1}$ Now at Laboratory for the Psychology of Child Development and Education, CNRS Unit 8240, Paris-Descartes University and Caen University, F-75005, Paris, France.
} 
spatial attention, studies are scarce. Jansen et al. (2004) compared functional transcranial Doppler sonography (fTCD) to fMRI measures during a landmark task in which subjects judged the accuracy of line bisections and demonstrated that both techniques were able to concordantly determine visuospatial hemispheric lateralization in healthy participants (Jansen et al., 2004).

To explore the factors that could explain the variability of spatial cerebral lateralization with fMRI, it is important to first verify whether the spatial attentional task fulfils several conditions in a sample of right-handed healthy participants.

A first condition that must be fulfilled is that the spatial attention task that is used to investigate spatial cerebral lateralization should elicit behavioural asymmetry. An example of a task that induces freeviewing behavioural asymmetries is the well-known "paper and pencil" line bisection task. This task requires the subject to mark the apparent midpoint of a long horizontal line drawn on a sheet of paper. Whereas some right hemispheric brain-injured patients bisect lines far to the right of the true midline (Doricchi and Angelelli, 1999; Sperber and Karnath, 2016), neurologically intact subjects tend to systematically err to the left of centre, a phenomenon first referred by Bowers and Heilman as pseudoneglect (Bowers and Heilman, 1980). The misbisection to the left, although not as pronounced as the rightward bias observed in patients, appears to be a reliable phenomenon (Jewell and McCourt, 2000, see however Manning et al., 1990 for a report of important between-subjects variations in the direction of pseudoneglect). One hypothesis is that this leftward asymmetry may be the result of an attentional bias directed towards the left hemispace, arising from asymmetries in hemispheric activation, with a right hemisphere involvement in visuospatial attention (Kinsbourne, 1970; Mesulam, 1999; Nicholls and Roberts, 2002). Due to the constraint that the subject must remain still during fMRI scanning, the classical "paper and pencil" line bisection task is difficult to set up, so usually a perceptual variant, called the landmark task is used, where the subjects decide the accuracy of line bisections (Fink et al., 2002). In a previous study, we designed a line bisection judgement (LBJ) task during which the participant was asked to judge if a bisection mark was located at the centre of a horizontal line or if it slightly deviated to the left or right of the midline. A spatial deviation bias based on the errors was computed for each individual, and the results demonstrated that the subjects more frequently erroneously judged that the vertical segment was deviated to the left of the true bisection, consistent with pseudoneglect (Zago et al., 2015). In the present experiment, we modified both the stimuli and design to verify whether this error bias was robust and not linked to the experimental parameters.

Another point that needs to be investigated is whether the LBJ attentional bias is specific to the task or related to other attentional biases in healthy participants. For example, the cancellation task, where the participant is asked to mark target items in a cluttered array of distractors, is another popular paper-and-pencil method for assessing spatial neglect (Weintraub and Mesulam, 1985). Although this task also elicits a rightward behavioural bias in neglect patients, some studies have shown that these two biases may dissociate, with patients showing specific deficits in line bisection but not in cancellation and vice versa (Binder et al., 1992; Ferber and Karnath, 2001). In addition, some of the regions critical for accurate cancellation performance are not required for unbiased bisection (Rorden et al., 2006; but see Molenberghs and Sale, 2011). This dissociation has been confirmed in healthy people, with the engagement of different neural networks according to the type of attentional task (visual search vs. bisection) (Revill et al., 2011). Here, we used the "paper and pencil" symbol cancellation task (SCT) to evaluate the cancellation bias based on the centre of cancellation score (CoC, Rorden and Karnath, 2010) and investigated the relationship between both biases.

Furthermore, in accordance with the hypothesis that pseudoneglect is associated with a right hemispheric dominance for spatial attention (Bowers and Heilman, 1980), the behavioural bias might be related to the cerebral asymmetry found during the LBJ task. For example, Szczepanski and Kastner (2013) demonstrated brain-behaviour correlation such that an individual's behavioural spatial bias, as measured using the landmark task, can be predicted by the degree of lateralization of the frontoparietal cortex (Szczepanski and Kastner, 2013). We also found such a relation between the LBJ bias and hemispheric lateralization in right- and left-handers (Zago et al., 2015). In the present study, we verified whether this association was also found in our sample of right-handers and whether it was specific to LBJ or related to the $\mathrm{CoC}$ measured during the "paper and pencil" cancellation task. In addition, one of the critical questions in cognitive neuroscience is how the variability in the functional organization of the human brain affects cognitive performance. In the language domain, it has been shown that, although healthy adults weakly lateralized for language performed lower on language or visuospatial tests than typical leftlateralized subjects, no correlation existed between the cerebral lateralization for language and performance (Mellet et al., 2014). In contrast, in children, some studies have demonstrated an association between the strength of cerebral lateralization and performance in language and visuospatial domains (Everts et al., 2009; Groen et al., 2012). Here, we assessed whether spatial cerebral lateralization could be linked to visuospatial skills in adults.

The spatial attention task should produce functional asymmetries linked to spatial attention processes involved in the task and should not be driven by other parameters that are known to elicit asymmetries, such as motoric responses, eye movement activity and visual attention shifting (Petit et al., 2014; Petit et al., 2009; Shulman et al., 2010). Consequently, the spatial attentional task should be compared to a control task that controls for low level perceptual activities and eye and hand motor activities performed during the attentional task. In addition, these functional asymmetries should be evaluated by statistical methods, using the reliable laterality index (LI) that provides quantitative information about hemispheric lateralization (Wilke et al., 2007) and voxel-wise comparisons of the magnitude of activation in the two hemispheres to yield a statistically valid regional asymmetric pattern (Liégeois et al., 2002; Stevens et al., 2005; Zago et al., 2015). Although they did not include such analyses, the first studies that used the landmark task in right-handers evidenced the involvement of a large network, including the frontal, parietal, temporal and occipital areas, in which activation tended to be predominant in the right hemisphere (Ciçek et al., 2009; Fink et al., 2000, 2001).

Finally, another important condition that the spatial task should fulfil is that the pattern of regional fMRI asymmetry observed during LBJ in healthy participants should be consistent with the pattern of lesions of neurological stroke patients who exhibit bisection bias (Corbetta et al., 2005; Rorden et al., 2006). Here, we investigated this issue by comparing the asymmetric brain regions of healthy participants to the brain lesions of stroke patients showing line bisection deficits, as reported by Rorden et al. (2006).

The aim of the present study was to validate the use of the LBJ task to adequately investigate the lateralized cerebral bases of spatial attention. We assessed 1) whether the LBJ task elicited a behavioural spatial attention bias and whether this relationship was observed with another attentional deviation bias, such as cancellation bias; 2) whether the LBJ task produced reliable cerebral functional asymmetries related to LBJ mechanisms, as assessed by the hemispheric lateralization index (HFLI) and regional functional asymmetry analyses computed from BOLD-fMRI images acquired during a LBJ task compared to a visuomotor control task; 3) whether the spatial cerebral asymmetry was related to the LBJ behavioural bias and whether this relationship was specific to LBJ or also found with cancellation bias, and we also explored the association between LBJ cerebral lateralization and visuospatial skills; 4) and whether the functional asymmetry pattern measured during the LBJ task was consistent with the lesion map of neurological stroke patients showing deficits regarding line bisection compared to patients without such a deficit (Rorden et al., 
2006).

A second objective of this study was to re-test the absence of the association between the cerebral lateralization for language and for spatial processing that we previously reported in right-handers who performed a language production task and LBJ task (Zago et al., 2015). Here, we verified whether this absence of a relation between cerebral lateralization was also observed with another component of language, such as speech listening (Tzourio et al., 1998), which, as the LBJ task, puts more demand on perceptual processing within each domain.

\section{Material and methods}

\subsection{Participants}

Fifty-one right-handed healthy volunteers (25 women, 26 men) as assessed using the Edinburgh handedness questionnaire $(+90 \pm 15$; mean \pm SD; Oldfield, 1971) were included in the present study. The mean age of the entire sample was 28 years ( $\mathrm{SD}=7$ years). All gave their informed, written consent and received an allowance for their participation. A local Ethics Committee (CCPRB Basse-Normandie, France) approved the experimental protocol. All subjects were free of brain abnormalities, which were verified by a trained neuroradiologist who evaluated their structural T1-MRI scans, and all had normal or corrected-to-normal vision. These participants also completed a series of behavioural tests in different sessions to evaluate their visuospatial abilities.

\subsection{FMRI tasks}

All participants performed an fMRI spatial attention task to evaluate cerebral lateralization for spatial attention. Forty-four participants completed a speech listening task in the same fMRI session to evaluate language hemispheric lateralization.

\subsubsection{Line bisection judgement (LBJ)}

Lateralization for spatial attention was examined based on a line bisection judgement task (LBJ) compared to a visuomotor control task. The paradigm consisted of four 30-sec blocks of LBJ alternated with four 30-sec blocks of control task (Fig. 1). During the LBJ, white horizontal line was bisected by a short vertical line and presented on a blank screen for $1.5 \mathrm{~s}$ with an inter-stimulus interval of $500 \mathrm{~ms}$. Participants were asked to judge if the bisection mark was placed at the centre of the horizontal line or if it slightly deviated to the left or right of the midline. They responded by pressing a one of three response buttons on a pad, with the right index finger corresponding to the answer "left", the right middle finger for "middle", and the right ring finger for "right". The horizontal lines were of five different lengths, subtending a visual angle of $6-10^{\circ}$, and were randomly positioned on

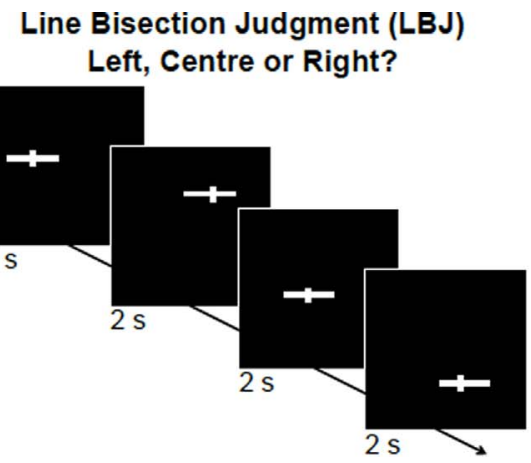

the screen. For left- and right-bisected trials, the vertical bisection mark was deviated by $0.2^{\circ}$ or $0.3^{\circ}$ of visual angle of the midline. Over the four blocks, 60 stimuli were presented with an equal number of centre-, leftward-, and rightward-bisected trials. For each participant, the response time (RT) and accuracy were recorded for each trial.

The control condition (Control) was designed to control for eye movements and visual attention shifting, low-level perceptual and motor response components of the LBJ task. Subjects performed saccadic eye movements at the same frequency than during the LBJ task $(0.5 \mathrm{~Hz})$ towards a visually presented dot and simultaneously pressed the response pad with the right index finger at each dot movement. The positions of the dot on the screen matched the positions of the bisection marks presented during the previous LBJ block. Prior to the scanning, participants were given a practice version of the task with 2 LBJ and 2 control blocks to become familiarized with the task.

\subsubsection{Story listening task}

To evaluate the hemispheric lateralization of language, we used a story-listening task that alternated $30 \mathrm{~s}$ blocks of a narrative spoken in the subjects' mother tongue (French) with $30 \mathrm{~s}$ blocks of a narrative in an unknown language (Tamil). Participants were in a dark room and were instructed to listen attentively to the stimuli while keeping their eyes closed. The story described a sports competition involving interactions between the characters. This paradigm has been shown to elicit reproducible leftward hemispheric asymmetries of lexicosyntactic areas (Tzourio-Mazoyer et al., 2010).

\subsection{Behavioural data}

\subsubsection{Spatial Attentional biases for the LBJ and Symbol Cancellation Test (SCT)}

2.3.1.1. LBJ. A spatial attentional bias was derived from errors performed during LBJ. This individual spatial attentional bias included the number of incorrect responses deviated to the left or to the right during the LBJ task. The number of leftward-deviated responses corresponded to the number of responses for which the subject responded "left" or "middle" for right bisected trials and "left" for middle bisected trials. The number of rightward responses included the number of responses for which the subjects responded "right" or "middle" for left bisected trials and "right" for middle bisected trials. The error deviation bias corresponded to the difference between the number rightward responses and the number of leftward responses. A negative value indicates that the subject produced more leftward errors.

2.3.1.2. SCT. In a different session, 47 out of 51 participants were

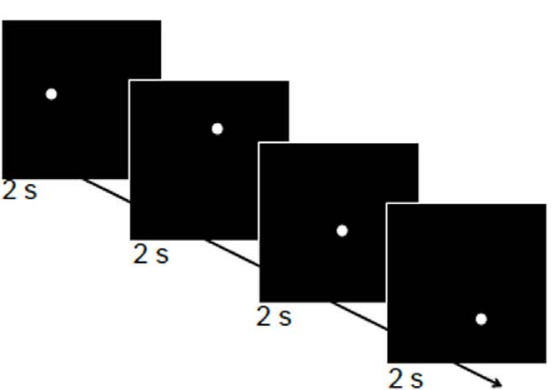

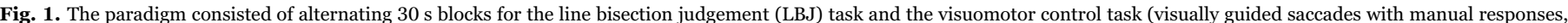

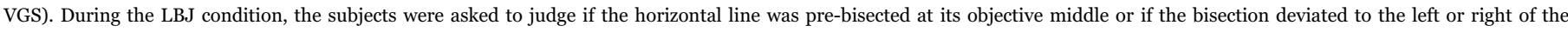

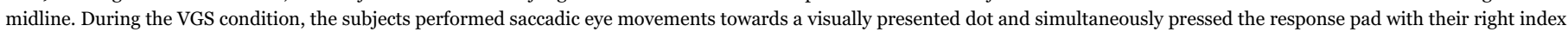
finger at each dot movement. Positions of the dot on the screen matched positions of the bisection marks presented during the previous LBJ block. 
asked to perform a paper and pencil Symbol Cancellation Test (SCT), which, in addition to the line bisection test, is another test used to investigate spatial neglect (Mesulam, 1985; Weintraub and Mesulam, 1985). To evidence a behavioural bias in healthy subjects, this test was time constrained, allowing $30 \mathrm{~s}$ for subjects to perform the cancellation. Using the software of Rorden and Karnath (2010), we computed for each participant a calibrated centre of cancellation (CoC) index that gives a value of attentional bias, with negative values indicating that the subject cancelled more targets on the left, consistent with leftward pseudoneglect.

\subsubsection{Visuo-spatial abilities}

A mean score of the visuospatial abilities for each participant was computed from the performance of the four visuo-spatial tests, namely the mental rotation test, which estimates the ability to rotate and spatially manipulate mental images (Vandenberg and Kuse, 1978); the Corsi Block Test, which evaluates visuospatial short-term memory (Della Sala et al., 1999); a home-made 3D maze test for evaluating topographic orientation skills (Mellet et al., 2013); and the Raven Matrix for assessing non-verbal reasoning (Raven, 1956).

\subsection{Image acquisition}

Imaging was performed using a Philips Achieva 3Tesla MRI scanner. Structural MRI protocols included a localizer scan, highresolution 3D T1-weighted volume acquisition $(\mathrm{TR}=20 \mathrm{~ms}$; $\mathrm{TE}=4.6 \mathrm{~ms}$; flip angle $=10^{\circ}$; inversion time $=800 \mathrm{~ms}$; turbo field echo factor $=65$; sense factor $=2$; matrix size $=256 \times 256 \times 180 ; 1 \mathrm{~mm}^{3}$ isotropic voxel size) and $\mathrm{T} 2{ }^{*}$-weighted multi-slice acquisition $\left(\mathrm{T}_{2}{ }^{*}\right.$-FFE sequence, $\mathrm{TR}=3500 \mathrm{~ms} ; \mathrm{TE}=35 \mathrm{~ms}$; flip angle $=90^{\circ}$; sense factor $=2 ; 70$ axial slices; $2 \mathrm{~mm}^{3}$ isotropic voxel size). Functional volumes were acquired using a whole-brain T2*-weighted echo-planar sequence (T2*-EPI, 240 volumes; $\mathrm{TR}=2000 \mathrm{~ms} ; \mathrm{TE}=35 \mathrm{~ms}$; flip angle $=80 ; 31$ axial slices; $3.75 \mathrm{~mm}^{3}$ isotropic voxel size) covering the same field of view as the $\mathrm{T}_{2}{ }^{*}$-FFE acquisition. The first four volumes of the sequence were discarded to allow for stabilization of the MR signal.

\subsection{Image analysis}

Preprocessing was performed based on Statistical Parametric Mapping subroutines (SPM5; http://www.fil.ion.ucl.ac.uk/spm). Anatomical T1-weighted volumes were spatially normalized by aligning individual anatomical volumes to specific cerebral tissue templates built from the $\mathrm{T} 1$ images of 80 right-handed subjects (40 men) acquired with the same scanner and acquisition parameters. Spatial normalization parameters were set to their SPM5 default values, providing for each subject a 3D, spatially normalized deformation field of T1 images mapped to the Montreal Neurological Institute (MNI) reference space. Each functional run was corrected for slice timing and motion and registered onto the $\mathrm{T}_{2}{ }^{*}$-FFE volume. Combining the $\mathrm{T}_{2}{ }^{*}$-FFE to T1-weighted registration parameters and the spatial normalization parameters, functional images were resampled to the $2 \times 2 \times 2 \mathrm{~mm}^{3}$ template space and spatially smoothed (Gaussian $6 \mathrm{~mm}$ full width at half maximum filter). First, for each participant, each block was modelled using a boxcar function of $30 \mathrm{~s}$ convolved with the canonical haemodynamic response function and its temporal derivative used in statistical parametric mapping (SPM). Low frequency confounds were excluded from the model by implementing a high-pass filter (120 s), and a movement regressor was also included in the model. First-level contrast images for each participant were computed (LBJ minus Control and French story minus Tamil story).

\subsection{HFLI analysis}

For each individual, hemispheric functional lateralization indices (HFLI) were computed using the LI-toolbox (Wilke and Schmithorst, 2006) with a bootstrapping method (Wilke et al., 2007) applied to activation minus Control t-maps for LBJ and story listening. This method involved the calculation of 20 equally sized thresholds from 0 to the maximum $t$ value. At each threshold, 100 bootstrapped samples with a resampling ratio of $\mathrm{k}=0.25$ were taken from the left and right hemispheres. All 10,000 possible HFLI combinations were then calculated from these samples for the surviving voxels on the left and the right using the formula $[(\mathrm{L}-\mathrm{R}) /(\mathrm{LR}+\mathrm{R})]$. The $25 \%$ highest and lowest values were excluded from the analysis (considered as outliers), and the remaining HFLI were averaged to compute an individual HFLI index. HFLI was computed within the anatomical template mask used for the fMRI data normalization, excluding the cerebellum. HFLI yielded values between -100 (complete right lateralization) and +100 (complete left lateralization).

\subsection{Voxel-based functional asymmetry analysis}

The functional asymmetry pattern of activation for LBJ was investigated by a voxel-based functional asymmetry analysis of the BOLD signal in SPM. This procedure, fully described in Liégeois et al. (2002), has been used elsewhere (Zago et al., 2015; Petit et al., 2009). Asymmetries were obtained by comparing the BOLD values of one hemisphere to those of the other hemisphere for each participant for LBJ minus Control. To do so, left/right flipped maps were computed (along the inter-hemispheric fissure; i.e., MNI stereotaxic $\mathrm{x}=0$ plane mirror images) resulting in individual BOLD and flipped-BOLD maps, and then compared. The asymmetry pattern was reported after applying a family-wise error (FWE) correction with $p<0.05$, and the peak coordinates are reported in Table 1 and illustrated in Fig. 2. Activation foci were labelled using automatic anatomical labelling software (AAL, Tzourio-Mazoyer et al., 2002).

Table 1

Asymmetry map during the LBJ task. The statistical threshold was set to $p<0.05$ and corrected for family-wise error (FWE).

\begin{tabular}{|c|c|c|c|c|c|c|}
\hline \multirow[t]{2}{*}{ Cluster size $\left(\mathrm{mm}^{3}\right)$} & \multirow[t]{2}{*}{ Localization } & \multicolumn{3}{|c|}{ MNI coordinates } & \multirow[t]{2}{*}{ T score } & \multirow[t]{2}{*}{$\%$ of cluster overlap with lesion map } \\
\hline & & $\mathrm{x}$ & $\mathrm{y}$ & $\mathrm{z}$ & & \\
\hline \multirow[t]{4}{*}{8411} & $\mathrm{R}$ intraoccipital sulcus/intraparietal sulcus & 32 & -68 & 34 & 12.2 & 41 \\
\hline & R middle occipital gyrus (MOG) & 36 & -64 & 20 & 8.3 & \\
\hline & R MOG & 38 & -82 & 4 & 7.4 & \\
\hline & $\mathrm{R}$ superior parietal gyrus & 30 & -62 & 56 & 5.4 & \\
\hline 2195 & $\mathrm{R}$ inferior frontal gyrus & 52 & 14 & 26 & 7.7 & 25 \\
\hline 1984 & L postcentral gyrus & -36 & -24 & 56 & 6.2 & 0 \\
\hline 938 & $\mathrm{R}$ medial superior frontal gyrus & 8 & 24 & 48 & 6.0 & 0 \\
\hline 673 & R temporal-occipital junction (TOJ) & 46 & -54 & -6 & 6.4 & 41 \\
\hline 331 & $\mathrm{R}$ posterior calcarine $(\mathrm{V} 1 \mathrm{p})$ & 16 & -90 & 2 & 6.4 & 0 \\
\hline 256 & $\mathrm{R}$ anterior insula & 36 & 24 & 6 & 5.6 & 93 \\
\hline
\end{tabular}



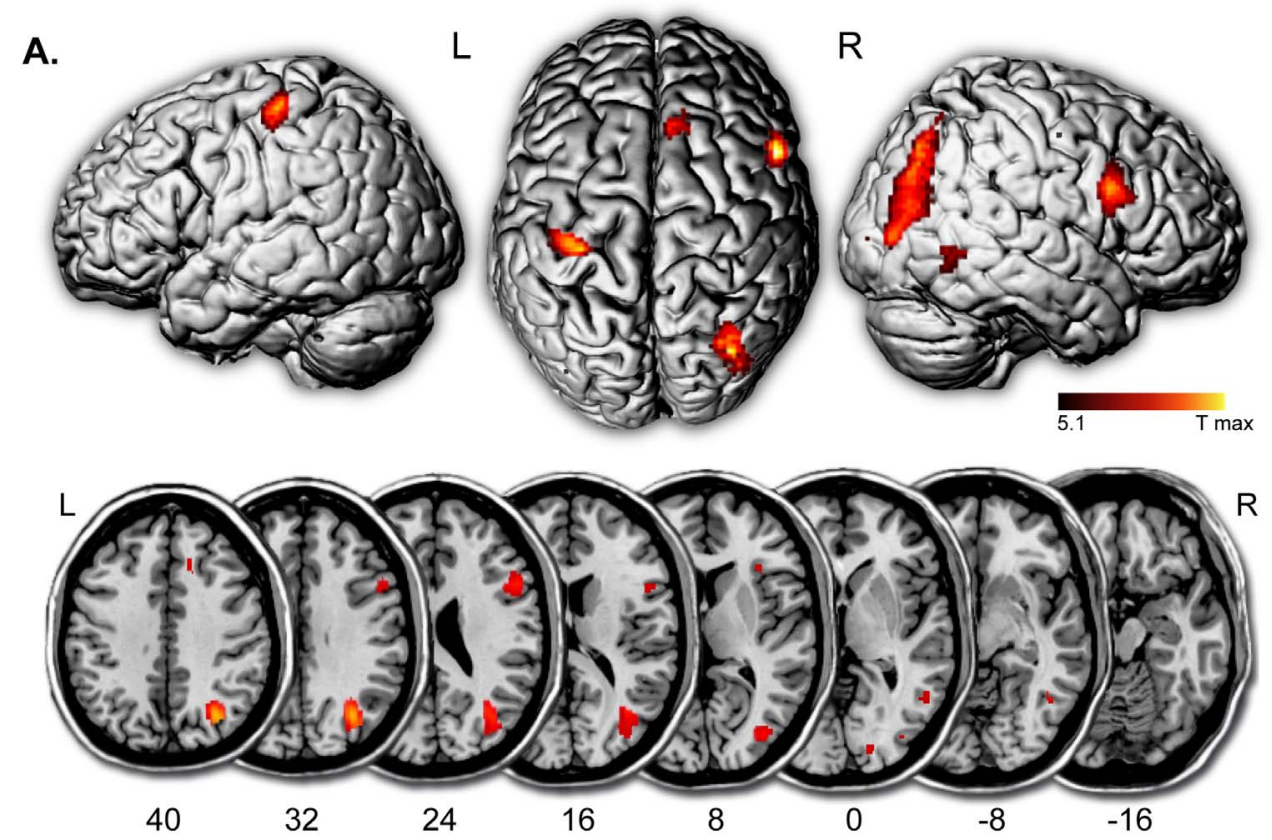

B.

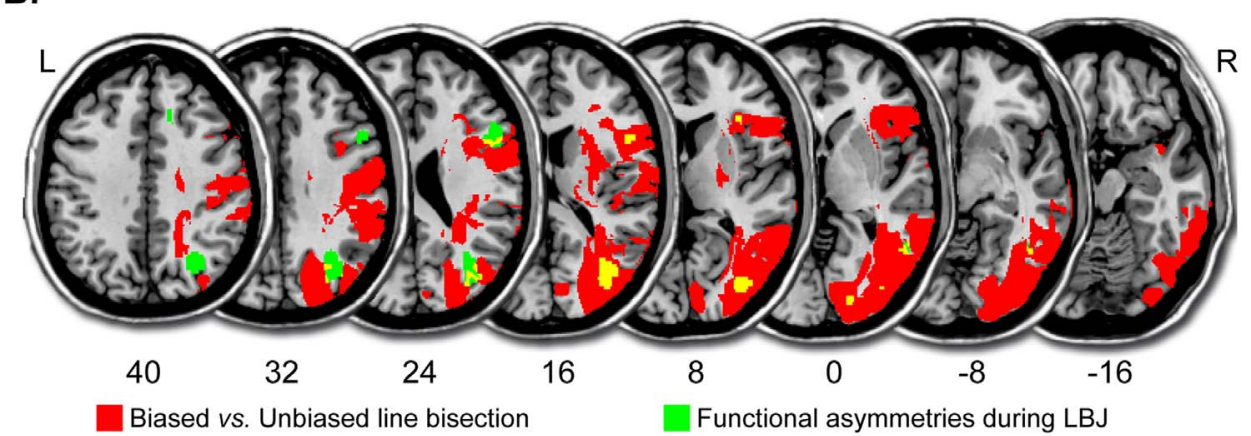

Overlap

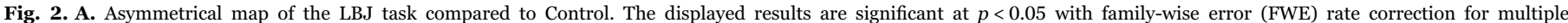

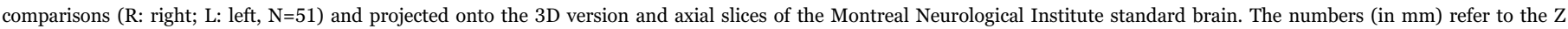

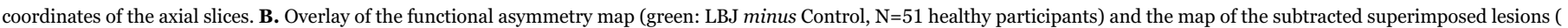

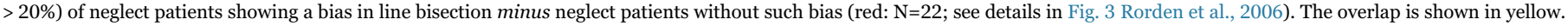
(For interpretation of the references to color in this figure legend, the reader is referred to the web version of this article).

\subsection{Overlay between functional LBJ asymmetry map of healthy subjects and line biased lesion map of patients}

To understand which regions could be those underlying the behavioural spatial bias during line bisection, we overlaid the functional asymmetry map obtained from our sample of healthy participants with the map of the subtracted superimposed lesions of biased minus unbiased line bisection stroke patients (Rorden et al., 2006). This latter map was thresholded to include the regions more frequently damaged ( $>20 \%$ ) in the biased versus the unbiased bisection patients (see details in Fig. 3 in Rorden et al., 2006). To quantify the overlap, we computed, for each asymmetric cluster, the percentage of overlap with the lesion map.

\subsection{Statistical analyses}

Unless otherwise specified, all statistical procedures were conducted using the JMP10 software package (www.jmp.com, SAS Institute Inc., 2012).

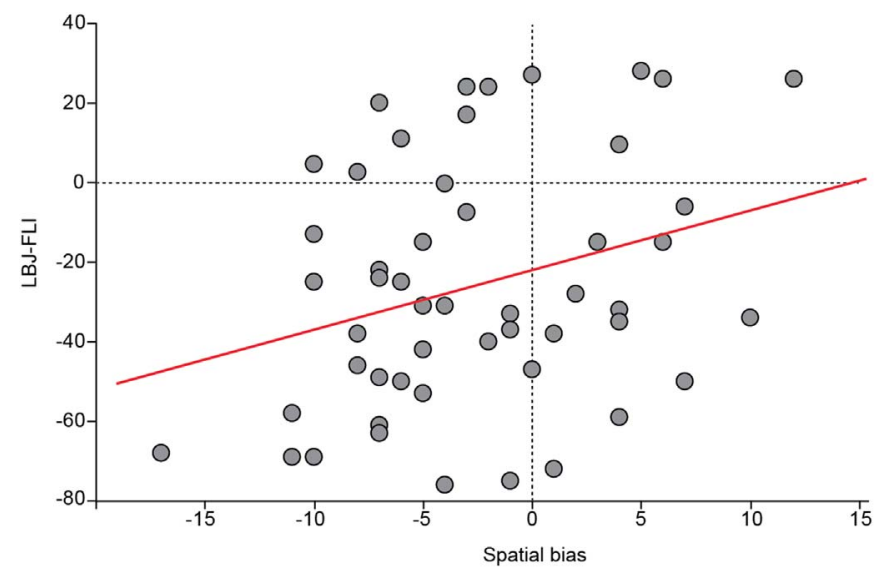

Fig. 3. Scatterplot of the relationship between the hemispheric lateralization index (LBJHFLI) and the LBJ deviation bias (spatial bias) based on errors.

\subsubsection{Behavioural data}

First, we verified whether attentional biases (LBJ error bias and $\mathrm{CoC}$ ) and visuospatial ability scores depended on sex, age, or educa- 
tional level (EL). Separate ANCOVAs were conducted with sex, age and EL as the between-subject factors for each variable. Then, we explored whether the two attentional biases were related to each other by performing a correlation analysis between the individual's biases of the 47 participants who had the two measures.

\subsubsection{Spatial attention and language comprehension HLIs}

To further investigate whether the lack of association between spatial and language production lateralization that we previously evidenced in right-handers (Zago et al., 2015) was also found for the language comprehension component, we performed a correlation analysis between spatial LBJ and the comprehension HFLI for the 44 right-handed individuals.

2.9.3. Relationships among spatial HFLI, attentional bias and visuospatial ability score

We investigated 1) whether cerebral lateralization was related to behavioural asymmetry as measured with the spatial error deviation bias; 2) whether this relationship can be extended to other attentional bias, such as the $\mathrm{CoC}$ measure resulting from the SCT; and 3) whether the degree of asymmetry in spatial attention was related to visuospatial skills. In a first ANCOVA analysis including all participants, we investigated the effect of the LBJ response bias and the visuospatial score on the LBJ HFLI. In the second analysis, which was performed on 47 participants, we explored the relation between the $\mathrm{CoC}$ and the LBJ HFLI. In both analyses, sex, age and EL were also included as covariates.

\section{Results}

\subsection{Spatial attentional biases}

\subsubsection{Line Bisection Judgement}

Performance was high during LBJ $(76.9 \pm 7.6 \%$ correct), and participants responded within an average of $1.0 \mathrm{~s}( \pm 0.1 \mathrm{~s})$. The error spatial attentional bias was negative on average (mean: -2.6 ; SD: 6.1, one sample $t$-test: $\mathrm{t}(50)=-3.09, \mathrm{p}=0.003$ ), indicating that participants produced more errors deviated to the left, consistent with a "pseudoneglect effect" (Bowers and Heilman, 1980). For the leftward errors, the condition that gathered most of the errors was when the subjects answered 'middle' for right-bisected trials $(6.3 \pm 3.6$; mean $\pm \mathrm{SD})$, while for the two others conditions, the number of errors was lower ('left' for middle-bisected trials: $1.4 \pm 1.4$ and 'left' for right-bisected trials: $0.2 \pm$ 0.5). For rightward errors, the same pattern was observed, with an increased number of errors corresponding to the participant selecting 'middle' for left-bisected trials $(4.49 \pm 3.14$; 'right' for middle-bisected trials: $0.58 \pm 0.93$; 'right' for left-bisected trials: $0.23 \pm 0.51$ ).

Attentional error bias did not differ between men and women $(\mathrm{F}(1,47)=1.8, \mathrm{p}=0.18)$ and was not dependent on the age $(\mathrm{F}(1,47)=1.9$, $\mathrm{p}=0.17)$ or educational level $(\mathrm{F}(1,47)=1.0, \mathrm{p}=0.30)$ of the participants.

\subsubsection{Symbol Cancellation Test}

On average, $48.4 \pm 6.7$ targets were cancelled in $30 \mathrm{~s}$ during the SCT. The mean CoC score was negative ( -0.07 ; SD: 0.10 , one sample $t$ test: $\mathrm{t}(46)=-5.2, \mathrm{p}<0.0001)$, indicating that participants cancelled more targets on the left than on the right of the space. This bias was consistent with a leftward "pseudoneglect effect" but, of course, could also be evoked by left-to-right reading and writing habits in our culture. The CoC score did not differ between men and women $(F(1,43)=2.9$, $\mathrm{p}=0.1)$ and was not dependent on the age $(\mathrm{F}(1,43)=0.04, \mathrm{p}=0.82)$ or educational level $(\mathrm{F}(1,43)=0.17, \mathrm{p}=0.98)$ of the participants.

The first analysis was designed to see if these two attentional biases, each exhibiting leftward bias, were related to one another. To accomplish this, we correlated the individual biases of the 47 participants for which we had both measures. The correlation was not significant $\left(\mathrm{R}^{2}=0.006, \mathrm{p}<0.58 ; \mathrm{t}=0.56\right)$.

\subsection{Visuospatial abilities}

The visuospatial score showed a classical effect of sex $(F(1,47)$ $=17.6, \mathrm{p}=0.0001$ ) with a better score for men than for women, an effect of $\mathrm{EL}(\mathrm{F}(1,47)=7.6, \mathrm{p}=0.008)$, with performances increasing with the year of schooling, and no effect of age $(F(1,47)=2.2, p=0.14)$.

\subsection{Relationship between language comprehension and spatial attention HFLIS}

For LBJ, the HFLI mean value was negative $(-26.1 \pm 31.0$; range: $[-76 ;+28])$, indicating a rightward hemispheric asymmetry during the LBJ task across the sample subjects (one-sample $t$-test, $\mathrm{t}(50)=6.0 \mathrm{p}<$ $0.0001)$. For story listening, the average HFLI value was positive (31.2 \pm 40 ; range: $[-79 ;+76])$, indicating a leftward hemispheric asymmetry. For the 44 participants that had both spatial and comprehension lateralization measures, we did not find a significant correlation between spatial and comprehension HLIs $\left(\mathrm{R}^{2}=0.0001, \mathrm{t}(44)=0.08\right.$, $\mathrm{p}=0.9$ ).

\subsection{Relationship between spatial HFLI, attentional biases and visuospatial score}

When behavioural data were confronted with spatial HFLI, a significant association was only found between spatial HFLI and LBJ error deviation bias $\left(\mathrm{F}(1,45)=4.5, \mathrm{p}=0.03\right.$, eta $\left.{ }^{2}=0.08\right)$. As illustrated in Fig. 3, the results indicated a positive correlation between HFLI and error bias (Pearson $\mathrm{r}=0.29 \mathrm{p}=0.03$ ), indicating that the strength of the right lateralization during the LBJ task increased with bias towards the left (i.e., the "pseudoneglect"). Note, however, that when the two extreme participants are removed, the correlation was still positive, but not significant $(t(49)=1.2 \mathrm{p}=0.2$; Pearson $\mathrm{r}=0.18)$. Finally, no effect of visuospatial score $(\mathrm{F}(1,45)=0.2, \mathrm{p}=0.6)$ or $\mathrm{CoC}(\mathrm{F}(1,42)=0.01$, $\mathrm{p}=0.9$ ) was found on the strength of the right lateralization.

\subsection{Voxel-based functional asymmetry}

As shown in Fig. 2A, the functional asymmetry analysis revealed important rightward asymmetries in a large posterior area encompassing the right posterior part of the intraparietal sulcus going downwards to the intraoccipital sulcus and laterally encompassing the middle occipital gyrus (MOG). Rightward asymmetries were also found in the posterior part of the calcarine sulcus (V1p) and in the temporooccipital junction (TOJ). In the frontal lobe, rightward asymmetries were found in the inferior frontal gyrus (opercular part), in the anterior insula and in the medial part of the superior frontal gyrus. Finally, a leftward asymmetry was detected in the postcentral gyrus corresponding to the motor response.

\subsection{Overlap between the functional LBJ asymmetry map and the lesion map of biased line bisection patients}

As shown in Fig. 2B, the overlapping areas of the maps are shown in yellow and include the MOG/intraoccipital sulcus, the TOJ, the inferior frontal and the anterior insula clusters. Specifically, for the MOG/ intraoccipital cluster, the overlap included $41 \%$ of the total cluster and was located in the lower part of the cluster, mainly in the MOG. For the TOJ cluster, the overlap also included $41 \%$ of the total cluster and was located in the most anterior part of the TOJ cluster. For the inferior frontal cluster, the overlap was in the inferior part (25\% of the total cluster), while the anterior insula cluster was entirely overlapped by the lesion map (93\%).

\section{Discussion}

The aim of the present study was to investigate whether the LBJ 
task met the conditions required to adequately investigate the lateralized cerebral bases of spatial attention and to be afterwards used to determine the factors of variability of spatial cerebral lateralization.

First, the LBJ task designed in the present experiment allowed for the assessment of behavioural attentional bias based on the errors that participants made to judge of the position of the vertical segment. Globally, participants made leftward-deviated errors. More specifically, they more often erroneously judged that the segment was deviated to the middle for lines that were bisected to the right. This bias is based on the number of deviated errors and thus differed from the millimetric distance of deviation from the midline traditionally measured in the "paper and pencil" line bisection. Although the error bias indicated a leftward asymmetry, similar to the paper and pencil line bisection measured in $\mathrm{mm}$, a main difference between the tests lies in the more important hand motor component in the classical "paper and pencil" test. Keeping this difference in mind, it is very likely, however, that the leftward deviation observed in LBJ and the pseudoneglect reported in the traditional line bisection task rely on the same attentional bias.

The leftward error bias observed in the present experiment was consistent with the spatial bias found in our previous neuroimaging study, using different stimuli, and with a larger population, including left-handers (Zago et al., 2015). In the previous study, LBJ stimuli were displayed at three different positions along the horizontal axis with three different lengths, and the bisection mark was deviated by $0.3^{\circ}$ to the left or right of the centre. Here, horizontal lines of five different lengths were randomly positioned on the screen (from 6 to $10^{\circ}$ of visual angle), and the bisection mark was deviated by $0.2^{\circ}$ or $0.3^{\circ}$ of the visual angle of the midline. The lengths of the lines were chosen to induce consistent leftward bias (Benwell et al., 2013). Among the 51 participants of the present study, 25 were also included in the study by Zago et al. (2015). For these participants, we compared the two spatial error biases. Although the design and stimuli were different, there was no difference between the bias values (paired- $t$-test $\mathrm{t}(24)=0.77, p=0.4$ ). Furthermore, both bias values were correlated $\left(R^{2}=0.31, t(24)=3.2\right.$, $p=0.003)$. Taken together, these findings suggest that the spatial attentional error bias obtained in this LBJ task is robust at the population level.

The present results demonstrated that both LBJ error bias and $\mathrm{CoC}$ elicited leftward behavioural biases. This indicates that both tasks are reliable in eliciting behavioural asymmetry in healthy subjects. However, these scores were not correlated in the 47 participants that had the two attentional measures. This absence of correlation has also been found in neglect patients (Binder et al., 1992; Ferber and Karnath, 2001; Sperber and Karnath, 2016), indicating separable components. Furthermore, no correlation between $\mathrm{CoC}$ and hemispheric lateralization was found, while the latter was associated with LBJ bias. This reinforces the hypothesis that both biases rely on different attentional mechanisms and that the cerebral lateralization of the neural networks reported in the present study tended to be more specific to the LBJ task. This is also in agreement with several brainlesion studies showing a dissociation of deficits between cancellation and line bisection tasks in neglect patients subtended by different anatomical correlates (Binder et al., 1992; Rorden et al., 2006).

The LBJ task elicited statistically reliable rightward functional asymmetries at both the hemispheric and regional levels. In particular, a large rightward asymmetry was found in a posterior brain region that included the posterior parietal cortex, the intraoccipital sulcus, and the MOG. Additional rightward asymmetries were detected in the posterior part of the calcarine fissure and in the inferior occipito-temporal region. The rightward asymmetry of the posterior occipito-parietal regions is in line with previous studies that used tasks that required the perception of object size or spatial judgements (Binder et al., 1992; Rorden et al., 2006), such as the landmark task or LBJ (BadzakovaTrajkov et al., 2011; Cai et al., 2013; Ciçek et al., 2009; Fink et al., 2001; Revill et al., 2011; Zago et al., 2015). The activity within the right inferior TOJ has been related to focused spatial attention mechanisms, specifically when attention is focused on a specific location to allow for the analysis of the visual stimulus (Corbetta and Shulman, 2002). This type of process is typically involved in LBJ. Accordingly, increased activation in the lateral TOJ has also been reported during a landmark task compared to a visual search task (Revill et al., 2011). In frontal regions, rightward asymmetries have been found in the inferior frontal gyrus, the anterior insula, and the medial frontal cortex. Their involvement could be related to non-spatial attentional processes required to perform LBJ compared to saccades. Such a hypothesis is in line with previous studies that have considered the inferior frontal region as involved in the executive control of attention (Bush et al., 2000; Fan et al., 2002). The involvement of these executive areas is likely related to the multiple response choices that the participants are faced with during LBJ. Accordingly, neglect patients with lesions involving the right inferior frontal region showed deficits in inhibiting responses to previously found targets during a visual search task, characterized by re-clicking old targets that they had visibly cancelled previously (Mannan et al., 2005). Finally, within this pattern of asymmetry, even if the motor activity was present during the visuomotor control, we detected a leftward functional asymmetry in the primary motor cortex. This leftward activity could correspond to the multiple-choice right-hand manual response during the LBJ task compared to the simple visuo-motor control task.

Another condition that the LBJ fulfilled is that the LBJ attentional bias was correlated with the degree of hemispheric lateralization. The more the individuals exhibited leftward pseudoneglect, the more they were rightward lateralized. This correlation indicated that the strength of rightward lateralization somehow predicts the degree of pseudoneglect. Note, however, that this effect is small, as only $8 \%$ of the variance is explained, and needs to be characterized using a large number of participants. For example, as shown in Fig. 3, the two extreme subjects for both LBJ bias and LBJ HFLI distributions, although not considered as statistical outliers, play a marked role in this correlation. When removed from the analysis, the correlation performed on the remaining 49 subjects remains positive, but did not reach significance. This indicates that the association between behavioural and cerebral biases is subtle and requires a large sample of subjects to explore the range of inter-individual variability for each of the behavioural and cerebral biases. Specifically, we previously showed this association with a larger sample of 293 subjects including right- and left-handers (Zago et al., 2015).

Although the neural origin of pseudoneglect is not resolved, previous studies have shown a correlation between behavioural bias and regional brain activity. For example, Benwell et al. (2014) demonstrated a positive correlation (Pearson $\mathrm{r}=0.544, p=0.024$ ) between left behavioural bias strength and right asymmetry in the right temporo-parietal junction (TPJ) using EEG (Benwell et al., 2014). Szczepanski and Kastner (2013) found a correlation $(\mathrm{r}(10)=-0.71, p<$ 0.05; in Szczepanski and Kastner, 2013) in the dorsal frontoparietal network, for which subjects with stronger LI values in the left hemisphere of this network tended to show a bias towards the right visual field while performing a landmark task, and those with stronger LI values on average in the right hemisphere tended to show a bias towards the left hemi-field. Here, to specifically explore this point, we computed lobar LIs using the pre-defined frontal, parietal, temporal and occipital lobes of the LI toolbox (Wilke and Lidzba, 2007). To investigate the effect of spatial bias on lobar LIs, we performed a MANOVA with the same factors as before namely, sex, age, EL, LBJ bias and visuospatial score. It indicated a main effect of LBJ bias across lobes $(\mathrm{F}(1-45)=6.2 p=0.01)$, with no specific effect of LBJ bias on a given lobe (Bias $\mathrm{x}$ Lobes, $p=0.9$ ). This global result can be interpreted differently: either the brain/behavioural relationship observed at the hemispheric level is not restricted to a specific lobe, or the association observed the hemispheric level requires an increased number of subjects to be evidenced at the regional level. In accordance with this latter interpretation, our previous study including a large number of 
subjects with large inter-individual variability showed that this association between the degree of rightward lateralization and pseudoneglect observed at the hemispheric level was found in the frontal lobe but not in the occipital lobe (Zago et al., 2015). Furthermore, additional correlational studies are required to elucidate the relationships between behavioural biases, either measured by the classical paper and pencil line bisection task (motor bias) or by perceptual landmark tasks or LBJ (perceptual bias), and whole-brain voxel-based asymmetry maps in a large sample of subjects.

While associated with LBJ bias, the strength of the rightward lateralization during LBJ was not related with visuo-spatial ability. In the language domain, it has also been shown that although participants who exhibited weak lateralization for language performed lower on language or visuospatial tests than typically left-lateralized subjects, no correlation existed between the HFLI and performance (Mellet et al., 2014). The present outcome extends this result in showing that no linear relationship exists between the spatial attentional HFLI measured during LBJ and performance in the spatial domain in adults, although such a relation has been reported in children (Everts et al., 2009).

The last condition that the LBJ should fulfil is a consistency of brain regions, revealing a rightward asymmetry in healthy participants and revealing line bisection deficits in stroke patients, as observed in the study by Rorden et al. (2006). The results demonstrated that the overlap between the clusters of asymmetry of healthy participants and the lesion map of line bisection neglect patients revealed a network of regions, including the occipito-temporal (MOG and TOJ) regions and the inferior frontal/anterior insula regions. For the posterior brain regions, this overlap may indicate that the line bisection bias strongly relies on visual processing (Revill et al., 2011; Sperber and Karnath, 2016). For LBJ, this might be related to the increased visual processing required to perform visual judgement and the reduced motor component compared to the traditional paper and pencil task. Regarding the frontal part, recent studies have shown segregated functions within the anterior insula and demonstrated a role of the right anterior insula in processes related to task-level control and focal attention (Nelson et al., 2010) and to attention and processing speed (Touroutoglou et al., 2012). Interestingly, Umarova et al. (2010) showed that these regions are structurally connected with ventral trajectories in the right hemisphere in healthy subjects (Umarova et al., 2010). In the present experiment, these potentially connected overlapping regions might subtend the visual bias found both in healthy participants and stroke patients. Future investigations are, however, required to investigate the question of the specificity of these regions at the origin of LBJ behavioural bias.

Finally, the results showed that there was no association between the strengths of lateralization between spatial LBJ and language comprehension lateralization. This finding extends our previous work on the absence of an association between language production and spatial attention in right-handers (Zago et al., 2015) to the comprehension component of language. The results of these two studies suggest that this independence between production and comprehension language processes and spatial hemispheric lateralization seems to be the overall pattern of brain organization across cognitive functions in righthanders.

In conclusion, based on various criteria, the present study demonstrated that the LBJ task is adequate to reveal cerebral lateralization of spatial attention mechanisms involved in line bisection. It can be reliably used to explore the variability of spatial attention hemispheric specialization.

\section{References}

Badzakova-Trajkov, G., Häberling, I.S., Corballis, M.C., 2011. Magical ideation, creativity, handedness, and cerebral asymmetries: a combined behavioural and fMRI study. Neuropsychologia 49 (10), 2896-2903. http://dx.doi.org/10.1016/ j.neuropsychologia.2011.06.016.

Benwell, C.S., Harvey, M., Thut, G., 2014. On the neural origin of pseudoneglect: EEGcorrelates of shifts in line bisection performance with manipulation of line length. Neuroimage 86, 370-380.

Benwell, C.S.Y., Harvey, M., Gardner, S., Thut, G., 2013. Stimulus- and state-dependence of systematic bias in spatial attention: additive effects of stimulus-size and time-ontask. Cortex 49, 827-836.

Binder, J., Marshall, R., Lazar, R., Benjamin, J., Mohr, J.P., 1992. Distinct syndromes of hemineglect. Arch. Neurol. 49 (11), 1187-1194.

Binder, J.R., 2011. Functional MRI is a valid noninvasive alternative to wada testing. Epilepsy Behav. 20 (2), 214-222.

Bowers, D., Heilman, K.M., 1980. Pseudoneglect: effects of hemispace on a tactile line bisection task. Neuropsychologia 18 (4-5), 491-498.

Bush, G., Luu, P., Posner, M.I., 2000. Cognitive and emotional influences in anterior cingulate cortex. Trends Cogn. Sci. 4 (6), 215-222.

Cai, Q., Van der Haegen, L., Brysbaert, M., 2013. Complementary hemispheric specialization for language production and visuospatial attention. Proc. Natl. Acad. Sci. USA 110 (4), 322-330. http://dx.doi.org/10.1073/pnas.1212956110.

Ciçek, M., Deouell, L.Y., Knight, R.T., 2009. Brain activity during landmark and line bisection tasks. Front. Hum. Neurosci. 3, 7. http://dx.doi.org/10.3389/ neuro.09.007.2009.

Coppens, P., Hungerford, S., Yamaguchi, S., Yamadori, A., 2002. Crossed aphasia: an analysis of the symptoms, their frequency, and a comparison with left-hemisphere aphasia symptomatology. Brain Lang. 83 (3), 425-463.

Corbetta, M., Shulman, G.L., 2002. Control of goal-directed and stimulus-driven attention in the brain. Nat. Rev. Neurosci. 3 (3), 201-215. http://dx.doi.org/ 10.1038/nrn755.

Corbetta, M., Kincade, M.J., Chris Lewis, C., Snyder, A.Z., Sapir, A., 2005. Neural basis and recovery of spatial attention deficits in spatial neglect. Nat. Neurosci. 8 (11), 1603-1610.

Della Sala, S., Gray, C., Baddeley, A., Allamano, N., Wilson, L., 1999. Pattern span: a tool for unwelding visuo-spatial memory. Neuropsychologia 37 (10), 118 9-119 9.

Doricchi, F., Angelelli, P., 1999. Misrepresentation of horizontal space in left unilateral neglect: role of hemianopia. Neurology 52, 1845-1852.

Dronkers, N.F., Knight, R.T., 1989. Right-sided neglect in a left-hander: evidence for reversed hemispheric specialization of attention capacity. Neuropsychologia 27 (5), $729-735$.

Dym, R.J., Burns, J., Freeman, K., Lipton, M.L., 2011. Is functional MR imaging assessment of hemispheric language dominance as good as the wada test?: a metaanalysis. Radiology 261 (2), 446-455. http://dx.doi.org/10.1148/radiol.11101344.

Everts, R., Lidzba, K., Wilke, M., Kiefer, C., Mordasini, M., Schroth, G., Perrig, W., Steinlin, M., 2009. Strengthening of laterality of verbal and visuospatial functions during childhood and adolescence. Hum. Brain Mapp. 30, 473-483.

Fan, J., McCandliss, B.D., Sommer, T., Raz, A., Posner, M.I., 2002. Testing the efficiency and independence of attentional networks. J. Cognit. Neurosci. 14 (3), 340-347. http://dx.doi.org/10.1162/089892902317361886.

Ferber, S., Karnath, H.-O., 2001. How to assess spatial neglect - Line bisection or cancellation tasks? J. Clin. Exp. Neuropsychol. 23 (5), 599-607.

Fink, G.R., Marshall, J.C., Weiss, P.H., Zilles, K., 2001. The neural basis of vertical and horizontal line bisection judgements: an fmri study of normal volunteers. NeuroImage 14 (1 Pt 2), S59-S67. http://dx.doi.org/10.1006/nimg.2001.0819.

Fink, G.R., Marshall, J.C., Weiss, P.H., Toni, I., Zilles, K., 2002. Task instructions influence the cognitive strategies involved in line bisection judgements: evidence from modulated neural mechanisms revealed by fMRI. Neuropsychologia 40 (2), 119-130.

Fink, G.R., Marshall, J.C., Shah, N.J., Weiss, P.H., Halligan, P.W., Grosse-Ruyken, M., Freund, H.J., 2000. Line bisection judgements implicate right parietal cortex and cerebellum as assessed by fMRI. Neurology 54 (6), 1324-1331.

Groen, M., Whitehouse, A.J., Badcock, N.A., Dorothy, V.M., Bishop, D.M., 2012. Does cerebral lateralization develop? A study using functional transcranial Dopple ultrasound assessing lateralization for language production and visuospatial memory. Brain Behav. 2 (3), 256-269.

Heilman, K.M., Watson, R.T., Valenstein, E., 1993. Neglect and related disorders. Clin. Neuropsychol. 3, 279-336.

Hervé, P.-Y., Zago, L., Petit, L., Mazoyer, B., Tzourio-Mazoyer, N., 2013. Revisiting human hemispheric specialization with neuroimaging. Trends Cognit. Sci. 17 (2), 69-80.

Jansen, A., Flöel, A., Deppe, M., van Randenborgh, J., Dräger, B., Kanowski, M., Knecht, S., 2004. Determining the hemispheric dominance of spatial attention: a comparison between fTCD and fMRI. Hum. Brain Mapp. 23 (3), 168-180. http://dx.doi.org/ $10.1002 / \mathrm{hbm} .20055$.

Jewell, G., McCourt, M.E., 2000. Pseudoneglect: a review and meta-analysis of performance factors in line bisection tasks. Neuropsychologia 38 (1), 93-110.

Josse, G., Tzourio-Mazoyer, N., 2004. Hemispheric specialization for language. Brain Res Brain Res. Rev. 44 (1), 1-12.

Karnath, H.O., Rorden, C., 2012. The anatomy of spatial neglect. Neuropsychologia 50 (6), 1010-1017. http://dx.doi.org/10.1016/j.neuropsychologia.2011.06.027.

Kinsbourne, M., 1970a. The Cerebral Basis of Lateral Asymmetries in Attention. in Acta Psychologica 33 Attention and Performance III. North-Holland Publishing Company, Amsterdam, 193-201.

Liégeois, F., Connelly, A., Salmond, C.H., Gadian, D.G., Vargha-Khadem, F., Baldeweg, T., 2002. A direct test for lateralization of language activation using fMRI: comparison with invasive assessments in children with epilepsy. NeuroImage 17 (4), $1861-1867$.

Mannan, S.K., Mort, D.J., Hodgson, T.L., Driver, J., Kennard, C., Husain, M., 2005 Revisiting previously searched locations in visual neglect: role of right parietal and 
frontal lesions in misjudging old locations as new. J. Cogn. Neurosci. 17 (2), 340-354. http://dx.doi.org/10.1162/0898929053124983.

Manning, L., Halligan, P.W., Marshall, J.C., 1990. Individual variation in line bisection: a study of normal subjects with application to the interpretation of visual neglect. Neuropsychologia 28 (7), 647-655.

Mazoyer, B., Zago, L., Jobard, G., Crivello, F., Joliot, M., Perchey, G., Mellet, E., Petit, P., Tzourio-Mazoyer, N., 2014. PlosOne 9 (6), e101165.

Mellet, E., Jobard, G., Zago, L., Crivello, F., Petit, L., Joliot, M., Mazoyer, B., TzourioMazoyer, N., 2013. Relationships between hand laterality and verbal and spatial skills in 436 healthy adults balanced for handedness. Later.: Asymmetries Body Brain Cognit.. http://dx.doi.org/10.1080/1357650X.2013.796965.

Mellet, E., Zago, L., Jobard, G., Crivello, F., Petit, L., Joliot, M., Mazoyer, B., TzourioMazoyer, N., 2014. Weak language lateralization affects both verbal and spatial skills: an fMRI study in 297 subjects. Neuropsychologia 65, 56-62.

Mesulam, M.M., 1999. Spatial attention and neglect: parietal, frontal and cingulate contributions to the mental representation and attentional targeting of salient extrapersonal events. Philos. Trans. R. Soc. Lond. Ser. B Biol. Sci. 354 (1387), 1325-1346. http://dx.doi.org/10.1098/rstb.1999.0482.

Mesulam, M.M. (Ed.), 1985. Contemporary Neurology Series. Philadelphia, Davis, vol. 26.

Molenberghs, P., Sale, M.V., 2011. Testing for spatial neglect with line bisection and target cancellation: Are both tasks really unrelated? PLOS One 6 (7).

Nelson, S.M., Dosenbach, N.U., Cohen, A.L., Wheeler, M.E., Schlaggar, B.L., Petersen, S.E., 2010. Role of the anterior insula in task-level control and focal attention. Brain Struct. Funct. 214, 669-680.

Nicholls, M.E., Roberts, G.R., 2002. Can free-viewing perceptual asymmetries be explained by scanning, pre-motor or attentional biases? Cortex 38 (2), 113-136.

Oldfield, R.C., 1971. The assessment and analysis of handedness: the Edinburgh inventory. Neuropsychologia 9 (1), 97-113.

Petit, L., Zago, L., Vigneau, M., Andersson, F., Crivello, F., Mazoyer, B., Tzourio-Mazoyer, N., 2009. Functional asymmetries revealed in visually guided saccades: an FMRI study. J. Neurophysiol. 102 (5), 2994-3003. http://dx.doi.org/10.1152/ jn.00280.2009.

Petit, L., Zago, L., Mellet, E., Jobard, G., Fabrice Crivello, F., Joliot, M., Mazoyer, B., Tzourio-Mazoyer, N., 2014. Strong rightward lateralization of the dorsal attentional network in left-handers with right sighting-eye: an evolutionary advantage. Hum. brain Mapp. (2014). http://dx.doi.org/10.1002/hbm.22693.

Raven, J.C., 1956. Guide to the Standard Progressive Matrices. The Psychological Corporation, New York.

Revill, K.P., Karnath, H.O., Rorden, C., 2011. Distinct anatomy for visual search and bisection: a neuroimaging study. NeuroImage 57 (2), 476-481. http://dx.doi.org/ 10.1016/j.neuroimage.2011.04.066.

Rorden, C., Karnath, H.O., 2010. A simple measure of neglect severity. Neurospychologia $48,2758-2763$

Rorden, C., Fruhmann Berger, M., Karnath, H.O., 2006. Disturbed line bisection is associated with posterior brain lesions. Brain Res. 1080 (1), 17-25. http:// dx.doi.org/10.1016/j.brainres.2004.10.071.

Shulman, G.L., Pope, D.L., Astafiev, S.V., McAvoy, M.P., Snyder, A.Z., Corbetta, M., 2010. Right hemisphere dominance during spatial selective attention and target detection occurs outside the dorsal frontoparietal network. J. Neurosci. 30 (10), 3640-3651. http://dx.doi.org/10.1523/JNEUROSCI.4085-09.2010.

Sperber, C., Karnath, H.-O., 2016. Diagnostic validity of line bisection in the acute phase of stroke. Neuropsychologia 82, 200-204.

Stevens, M.C., Calhoun, V.D., Kiehl, K.A., 2005. Hemispheric differences in hemodynamics elicited by auditory oddball stimuli. NeuroImage 26 (3), 782-792. http://dx.doi.org/10.1016/j.neuroimage.2005.02.044.

Suchan, J., Karnath, H.-O., 2011. Spatial orienting by left hemisphere language areas: a relict from the past? Brain: J. Neurol. 134, 3059-3070.

Szczepanski, S.M., Kastner, S., 2013. Shifting attentional priorities: Control of spatial attention through hemispheric competition. J. Neurosci. 33 (12), 5411-5421. http://dx.doi.org/10.1523/JNEUROSCI.4089-12.2013.

Touroutoglou, A., Hollenbeck, M., Dickerson, B., Feldman Barrett, L., 2012. Dissociable large-scale networks anchored in the right anterior insula subserve experience and attention. Neuroimage 60, 1947-1958.

Tzourio, N., Crivello, F., Mellet, E., Nkanga-Ngila, B., Mazoyer, B., 1998. Functional anatomy of dominance for speech comprehension in left handers vs right handers. NeuroImage 8 (1), 1-16. http://dx.doi.org/10.1006/nimg.1998.0343.

Tzourio-Mazoyer, N., Petit, L., Razafimandimby, A., Crivello, F., Zago, L., Jobard, G., et al., 2010. Left hemisphere lateralization for language in right-handers is controlled in part by familial sinistrality, manual preference strength, and head size. J. Neurosci. 30 (40), 13314-13318.

Tzourio-Mazoyer, N., Landeau, B., Papathanassiou, D., Crivello, F., Etard, O., Delcroix, N., Joliot, M., 2002. Automated anatomical labeling of activations in SPM using a macroscopic anatomical parcellation of the MNI MRI single-subject brain. NeuroImage 15 (1), 273-289. http://dx.doi.org/10.1006/nimg.2001.0978.

Umarova, R., Saur, D., Schnell, S., P., Kaller, C., Vry, M.-S., Glauche, V., Rijntjes, M., Hennig, J., Kiselev, V., Weiller, C., 2010. Structural connectivity for visuospatial attention: significance of ventral pathways. Cereb. Cortex 20, 121-129.

Vandenberg, S., Kuse, A., 1978. Mental rotations, a group test of three-dimensional spatial visualization. Percept. Motor Skills 47, 599-604.

Wada, J., Rasmussen, T., 1960. Intracarotid injection of sodium amytal for the lateralization of cerebral speech dominance. J. Neurosurg. 17, 266-282.

Weintraub, S., Mesulam, M.M., 1985. In: Davis Company, F.A. (Ed.), Mental State Assessment of Young and Elderly Adults in Behavioral Neurology, Principles of Behavioral Neurology. Mesulam MM, Philadelphia, 71-123.

Wilke, M., Schmithorst, V.J., 2006. A combined bootstrap/histogram analysis approach for computing a lateralization index from neuroimaging data. NeuroImage 33 (2), 522-530. http://dx.doi.org/10.1016/j.neuroimage.2006.07.010.

Wilke, M., Lidzba, K., 2007. A new toolbox to assess lateralization in functional MR-data. J. Neurosci. Methods 163, 128-136.

Wilke, M., Krägeloh-Mann, I., Holland, S.K., 2007. Global and local development of gray and white matter volume in normal children and adolescents. Exp. Brain Res. 178 (3), 296-307. http://dx.doi.org/10.1007/s00221-006-0732-z.

Zago, L., Petit, L., Mellet, E., Jobard, G., Crivello, F., Joliot, M., Tzourio-Mazoyer, N., 2015. The association between hemispheric specialization for language production and for spatial attention depends on left-hand preference strength. Neuropsychologia. http://dx.doi.org/10.1016/j.neuropsychologia.2015.11.018. 